\title{
Simulations of denuded-zone formation during growth on surfaces with anisotropic diffusion
}

\author{
C. Ebner, K.-B. Park, J.-F. Nielsen, and J. P. Pelz* \\ Department of Physics, The Ohio State University, Columbus, Ohio 43210, USA
}

(Received 26 April 2003; published 9 December 2003)

\begin{abstract}
We have investigated the formation of denuded zones during epitaxial growth on surfaces exhibiting anisotropic diffusion of adparticles, such as $\mathrm{Si}(001)-2 \times 1$, using Monte Carlo simulations and a continuum model. In both the simulations, which were mainly for low-temperature cases (small critical clusters), and the continuum model, appropriate for high-temperature cases (large critical clusters), it was found that the ratio of denuded-zone widths $W_{f}$ and $W_{s}$ in the fast- and slow-diffusion directions scales with the ratio $D_{f} / D_{s}$ of the diffusion constants in the two directions with a power of $1 / 2$, i.e., $W_{f} / W_{s} \approx\left(D_{f} / D_{s}\right)^{1 / 2}$, independent of various conditions including the degree of diffusion anisotropy. This supplies the foundation of a method for extracting the diffusion anisotropy from the denuded zone anisotropy which is experimentally measurable. Further, we find that unequal probabilities of a diffusing particle sticking to different types of step edges [e.g., $S_{A}$ and $S_{B}$ steps on $\mathrm{Si}(001)]$ does not affect the relation $W_{f} / W_{s} \approx\left(D_{f} / D_{s}\right)^{1 / 2}$ seriously unless the smaller of the two sticking probabilities is less than about 0.1 . Finally, we examined the relation between the number of steps and the number of sites visited in anisotropic random walks, finding it is better described by a crossover from one-dimensional to two-dimensional behavior than by scaling behavior with a single exponent. This result has bearing on scaling arguments relating denuded-zone widths to diffusion constants for anisotropic diffusion.

DOI: 10.1103/PhysRevB.68.245404

PACS number(s): 68.43.Jk, 68.55.Ac, 68.43.De
\end{abstract}

\section{INTRODUCTION}

Surface diffusion is a central kinetic factor in the evolution of surface morphology during growth and processing of materials. As semiconductor technology gives rise to ever smaller devices, the importance of surfaces plays a steadily expanding role. On many semiconductor surfaces, such as the technologically important $\mathrm{Si}(001)$ surface, diffusion is significantly anisotropic, giving rise to a need to understand both the degree and implications of such behavior. In this paper we present numerical simulations of a model of island nucleation and growth on a flat terrace. The model is constructed with the $\mathrm{Si}(001)$ surface subjected to a rain of $\mathrm{Si}$ atoms in mind. In addition, an analytic model of island nucleation on a terrace under the same circumstances is studied.

Earlier work on this topic includes the investigations of Mo et al. ${ }^{1-3}$ dealing with the low-temperature limit with a "critical cluster size" equal to 2 , i.e., conditions such that a stable island can nucleate by the meeting of just two diffusing "adparticles." We use the term adparticle here because the diffusing species on $\mathrm{Si}(001)$ could be a single $\mathrm{Si}$ atom ${ }^{1}$ or a Si dimer. ${ }^{3-7}$ Mo et al. ${ }^{1}$ suggested that the diffusion anisotropy $D_{f} / D_{s}$ (where $D_{f}$ and $D_{s}$ are the diffusion constants along the fast and slow directions, respectively) could be quantified by measuring the relative width of the fast and slow "denuded zones" $W_{f}$ and $W_{s}$, these being regions close to a step at a terrace boundary where relatively few islands nucleate when the step is oriented perpendicular to the fastand slow-diffusion directions, respectively. Recently, scanning tunneling microscopy (STM) has been used to directly monitor diffusion anisotropy by following the motions of individual diffusing adparticles at low growth temperatures. ${ }^{78}$ However, this technique is so far not applicable at higher temperatures where most semiconductor growth and processing is done and where diffusion is too rapid for existing STM's to follow. Hence it remains useful to study diffusion anisotropy indirectly through the investigation of denuded zones.

In this paper we reexamine the relation between diffusion anisotropy and the relative size of the denuded zones in the directions of fast and slow diffusion for the following three reasons.

First, in Refs. 1-3 it was argued that the denuded-zone width $W$ should be proportional to a power of the diffusion constant $D, W \propto D^{\alpha}$ where $\alpha=1 / 6$ for isotropic diffusion in two dimensions and $\alpha=1 / 4$ for one-dimensional or highly anisotropic two-dimensional diffusion. It was further suggested that $W_{f} / W_{s} \approx\left(D_{f} / D_{s}\right)^{\beta}$ where $\beta$ is the same as $\alpha$, i.e., $\beta$ should vary between $1 / 6$ in the isotropic limit and $1 / 4$ in the anisotropic limit. By contrast, our Monte Carlo (MC) simulations of diffusion in two dimensions in the smallcritical-cluster limit display an exponent $\beta$ close to $1 / 2$ independent of a wide variety of conditions including the degree of anisotropy of the diffusion. This is a significant difference, in particular for any attempt to determine the degree of anisotropy of the diffusion from measurements of the denuded zone widths.

Second, we have developed and numerically solved an analytic model of cluster nucleation based on the work of Theis and Tromp ${ }^{9}$ which is applicable in the hightemperature limit where the critical cluster size is much larger than 2, and which is relatively difficult to study with MC techniques because of the necessary size of the simulation cell and length of the simulations. This model also gives clearly an exponent $\beta$ close to $1 / 2$ but does not so far allow for the observation of the growth of clusters once nucleated. In addition we have done a few representative MC simulations in the large-critical-cluster regime, finding results consistent with an exponent $\beta \approx 1 / 2$. 
Third, we wished to revisit the scaling arguments used by Mo et al. ${ }^{1}$ to determine how denuded-zone widths scale with diffusion constant and diffusion anisotropy, and from which the exponents $\alpha=1 / 6$ for isotropic diffusion and $\alpha=1 / 4$ for highly anisotropic diffusion were predicted. These arguments, and later ones by Pimpinelli et al., ${ }^{10}$ were based in part on the assumption that the number of sites $N_{v}$ visited by a random walker as a function of the number of steps $N_{s}$ in the walk scales as $N_{v} \sim N_{s}^{d / 2}$, where the effective dimension $d$ varies from $d=2$ for isotropic diffusion to $d=1$ in the anisotropic limit. More precisely, both Mo et al. and Pimpinelli et al. used $N_{v} \sim(D \tau)^{d / 2}$ where $D$ is a diffusion constant. As pointed out by Mo et al., while this relation is strictly true in one-dimensional diffusion, in two dimensions the correct relation is $N_{v} \sim N_{s} / \ln \left(N_{s}\right)$. Their assumption is that the logarithmic correction is not important for large enough $N_{s}$. However, our simulations of the island nucleation and growth process consistently produced a value of $\alpha$ that is $10-15 \%$ larger than $1 / 6$ for isotropic diffusion in two dimensions. We argue that this discrepancy is a consequence of the logarithmic term in the relation between $N_{v}$ and $N_{s}$. More generally, for anisotropic diffusion we find that the dependence of $N_{v}$ and $D$ on $N_{s}$ is better described as a crossover behavior from strict one-dimensional (1D) to 2D diffusion (at a value of $N_{s}$ where the probability of the walker having taken one step in the slow direction approaches unity) rather than as true scaling behavior with an effective $d$ between 1 and 2 .

\section{SIMULATION MODEL AND RESULTS}

The primary purpose of these simulations is to study the dependence of the relative denuded-zone width $r_{w}=W_{f} / W_{s}$ on the diffusion-anisotropy ratio $r_{D}=D_{f} / D_{s}$. The model treats particles on a two-dimensional $N \times N$ square lattice, typically with $N=1000$. The particles are placed randomly on lattice sites at a steady rate $R$ particles per site, starting at time $t=0$ and continuing until time $t_{0}$ at which point no further particles are deposited. Thus the number of monolayers deposited is $f=R t_{0}$ and is typically on the order of 0.01 ML (monolayer). Once deposited, a particle engages in a random walk in which steps are made to nearest-neighbor lattice sites. The walks can be anisotropic in that the probability of a step in one direction (the fast direction), designated as the $x$ direction, may be greater than the probability of a step in the other direction (the slow direction) which is the $y$ direction.

The simplest scheme employed for the diffusion process is as follows: When walkers encounter each other, they join to form an immobile island. When a walker encounters an edge of the system, it is removed, and when a walker encounters an island, it is incorporated into that island. The walks are continued until no mobile particles are left. An island is treated as a point object, occupying a single lattice site. Variants of this scheme have been investigated. Specifically, an island may be given a finite extent, covering a number of lattice sites equal to the number of particles it contains. Simulations have been done to investigate the consequences of finite islands anisotropic in shape. In addi-



FIG. 1. The island densities (relative to the central density) in the fast and the slow directions are shown as a function of the distance from the edge of the cell for a case with $r_{D}=9$ and a typical $D_{f}$. The denuded-zone is wider in the fast direction.

tion we have studied the consequences of having a sticking coefficient $p$ smaller than unity for particles moving in the fast direction, meaning that when a particle encounters a wall, island, or another diffusing particle when moving in the fast direction, it adheres to that object with probability $p$.

For a given set of conditions, simulations were repeated 3000-10 000 times and the results averaged. The density of islands was computed and found to be essentially uniform throughout the middle of the terrace and to decrease monotonically to zero at the edges. The denuded-zone width was determined from the criterion that the island density be, at the edge of the denuded zone, $60 \%$ of its value at the center of the terrace. Other possible criteria were examined (such as an island density equal to $50 \%$ or $70 \%$ of its central value) and were found to be essentially equivalent as regards the relative size of the denuded zones when $p=1$. Typical island density profiles close to the edge of the terrace in the fast and slow directions are shown in Fig. 1 for a simulation with $r_{D}=9$. The abscissa measures the position in units of the terrace size and the ordinate shows the island density relative to the density at the center. The denuded zone is wider in the fast direction than in the slow direction.

Figure 2 displays the island density $n_{i}$ (islands per site) in the central region of the terrace as a function of $D_{f} ; D_{f}$ is expressed in arbitrary units such that $D_{f}=1$ corresponds to the smallest diffusion constant simulated. Results are shown for $r_{D}=1,4,9,99$, and 999; the island density increases with increasing $r_{D}$ at given $D_{f}$ and is typically of the order of $10^{-3}$. As $D_{f}$ increases, the results approach straight lines, as in the simulations of Mo et al. ${ }^{1}$ The slope (on a log-log plot) of the results for isotropic diffusion is about 0.30 at the largest $D_{f}$ simulated and appears to be approaching the asymptotic value of $1 / 3$ predicted in Ref. 1 . However, that prediction is based on the relation $N_{v} \sim N_{s}$ which neglects the logarithmic term; one should expect to find a slightly larger slope than $1 / 3$ unless the simulations are exceedingly long.

There are two important parameters in the simulations which may be taken as the ratio $Q_{f}$ of the fast-diffusion rate 


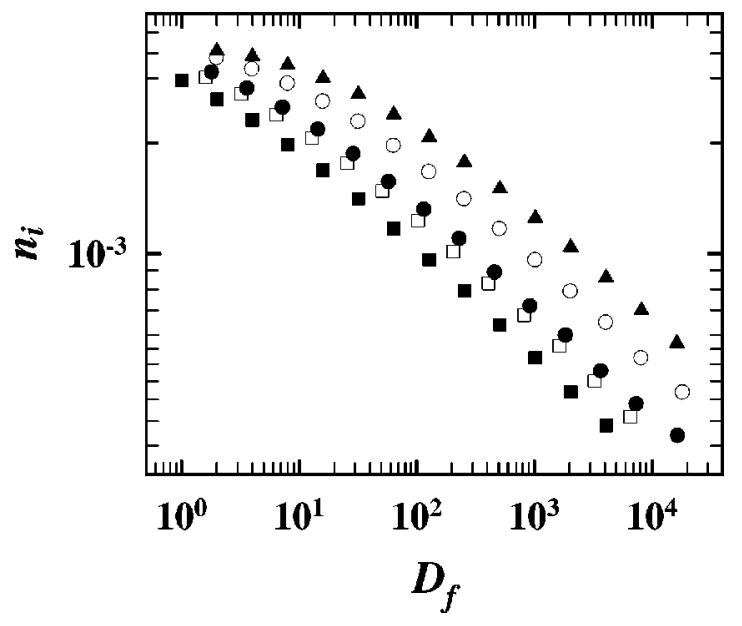

FIG. 2. The island density (islands/site) is plotted against $D_{f}$ (arbitrary units) for diffusion anisotropies $r_{D}$ of $1(\boldsymbol{\square}), 4(\square), 9$ (○), $99(\bigcirc)$, and $999(\boldsymbol{\Delta})$.

to the deposition rate, $Q_{f}=D_{f} / R$, and the diffusionanisotropy ratio $r_{D}=D_{f} / D_{s}$. In the simulations we fix $R$ and so $Q_{f} \propto D_{f}$. In the following we will describe results as functions of $r_{D}$ and $D_{f}$. We have typically done simulations using $1 \leqslant r_{D} \leqslant 10^{4}$ and $D_{f}$ with a relative range of $1: 512$. The limitations on the simulations are as follows: First, for small $D_{f}$ and/or large $r_{D}$, denuded-zone widths, especially in the slow direction, become very small, comparable to a lattice spacing, and, second, for large $D_{f}$, denuded-zone widths, especially in the fast direction, approach the size of the lattice. For a $1000 \times 1000$ lattice and an assumed $\mathrm{Si}(001)$ site spacing of $3.84 \AA$, the simulation cell corresponds to a terrace not much more than $1 / 3 \mu \mathrm{m}$ on a side, considerably smaller than the terraces in some experiments which display denuded zones of linear extent on the order of a several micrometers at higher temperatures; ${ }^{11}$ see, for example, Fig. 10. Also, the critical cluster size in the high temperature experiments is believed to be much larger than 2. Hence the simulations described here are most appropriate for comparison with lower-temperature experiments such as those of Mo et al. ${ }^{1-3}$ Nevertheless, one may expect that certain qualitative features of the observed behavior at higher temperatures, such as the manner in which the denuded-zone widths vary with $D_{f}$ and $r_{D}$, will be accurately reflected in the simulations.

Consider the dependence of $W_{f}$ on $D_{f}$ for fixed $r_{D}$. Figure 3 shows results for $W_{f} / N$ as a function of $D_{f}$. The different points are for $r_{D}$ equal to 1, 4, 9, 99, 999, and 9999; for given $D_{f}, W_{f}$ increases with increasing $r_{D}$.

The solid lines, included for reference purposes, have slopes (on a log-log plot) of $1 / 6$ and $1 / 4$, which describe the behavior predicted in Ref. 1 for isotropic $\left(r_{D}=1\right)$ and strictly $1 \mathrm{D}\left(r_{D} \rightarrow \infty\right)$ diffusion. Our simulation results for the isotropic system ( $\square$ ) fit quite well a line of slope 0.19 , almost $14 \%$ larger than $1 / 6$. As mentioned earlier, we believe the deviation of the simulation results from the prediction comes about because the latter assumes that $N_{v} \sim N_{s}$ in an isotropic two-dimensional random walk. The more correct relation $N_{v} \sim N_{s} / \ln \left(N_{s}\right)$ can be reasonably approximated as

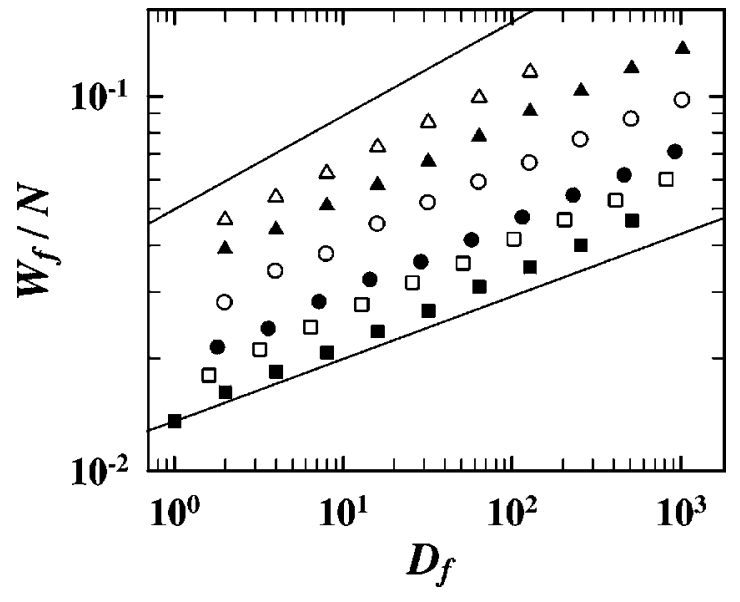

FIG. 3. The width of the denuded zone in the fast direction, in units of the total width of the system, is shown as a function of $D_{f}$ (arbitrary units) for diffusion anisotropies of $1(\boldsymbol{\square}), 4(\square), 9(\boldsymbol{O})$, $99(\bigcirc), 999(\boldsymbol{\Delta})$, and $9999(\triangle)$. Lines of slope (on a log-log plot) $1 / 6$ and $1 / 4$ are included for purposes of comparison.

$\propto N_{s}$ only if $N_{s}$ is very large. More precisely, one has from this relation a slope $m=d\left[\ln \left(N_{v}\right)\right] / d\left[\ln \left(N_{s}\right)\right] \approx 1-1 / \ln \left(N_{s}\right)$ which approaches unity very slowly as $N_{s}$ becomes large. For example, we see that $m$ varies from about 0.89 for $N_{s}$ $=10^{4}$ to just 0.95 even for $N_{s}=10^{8}$. We have made separate simulations of isotropic and anisotropic 2D random walks and found they are consistent with these estimates for $m$ in the isotropic case. If these values of $m$ are used in the dimensional argument of Ref. 1 , the result is $W_{f} \sim D_{f}^{\alpha}$ with $\alpha$ $\approx 0.186$ for $N_{s}=10^{4}$, and $\alpha \approx 0.175$ for $N_{s}=10^{8}$. The relevant question is, what is the typical number of steps a walker makes in the diffusion process before hitting another object? For an isotropic 2D walker this is roughly (but subject to the logarithmic correction) the inverse density of islands in units of islands per site. ${ }^{1}$ In our simulations the island density is typically in the vicinity of $10^{-3}$ as indicated by Fig. 2, implying that a diffusing particle must visit some $10^{3}$ sites before being absorbed into a cluster. That is a rather short walk, and $N_{v}$ is significantly less than proportional to $N_{s}$ which is reflected in the value of $\alpha$ extracted from the simulations.

In the case of highly anisotropic diffusion, e.g., $r_{D}$ $=9999$, one can see from Fig. 3 that the system does not yet behave as though the walk were strictly $1 \mathrm{D}$ in that the slope of $\log \left(W_{f}\right)$ against $\log \left(D_{f}\right)$ is still smaller than $1 / 4$. We have done separate simulations of a strictly 1D system and found that the results are best fit by a line of slope 0.256 which we regard as consistent with the prediction. We will return shortly to the question of why even the $r_{D}=9999$ case does not appear to quite reach the true 1D limit.

In previous studies, ${ }^{1,10}$ scaling arguments were made using an effective dimension $d$ between 1 and 2, whose precise value should be related to the degree of anisotropy. These arguments were based in large part on the assumption $N_{v}$ $\sim N_{s}^{d / 2}$ for an anisotropic 2D random walk. However, our simulations suggest that in fact the dependence of $N_{v}$ on $N_{s}$ for an anisotropic 2D random walk is better described by a crossover from strict 1D behavior $\left(N_{v} \sim N_{s}^{1 / 2}\right)$ for small $N_{s}$ to 




FIG. 4. The number of sites visited is plotted against the number of steps in anisotropic two-dimensional random walks with relative probability of steps in the $x$ and $y$ directions equal to $1(\triangle), 4(\mathbf{A})$, $9(\bigcirc), 99(\boldsymbol{O}), 999(\square)$, and $9999(\boldsymbol{\square})$. Lines of slope (on a $\log -\log$ plot) $1 / 2$ and 1 are included for comparison.

isotropic 2D behavior $\left[N_{v} \sim N_{s} / \ln \left(N_{s}\right)\right]$ for large $N_{s}$. In Fig. 4 we show $N_{v}$ vs. $N_{s}$ for walks with $r_{D}$ equal to (from top to bottom) 1, 4, 9, 99, 999, and 9999. Lines of slope (on a $\log -\log$ plot) $1 / 2$ and unity are included for reference purposes. One can see that for the isotropic case $\left(r_{D}=1\right)$ the slope (on a log-log plot) of the data is significantly less than unity for the largest $N_{s}$ shown (about $10^{6}$ ), which as discussed above is a consequence of the $\ln \left(N_{s}\right)$ term in the expression $N_{v} \sim N_{s} / \ln \left(N_{s}\right)$. One can also see that for the highly anisotropic walks, $N_{v}$ is roughly proportional to $N_{s}^{1 / 2}$ for small $N_{s}$, but eventually changes to essentially isotropic $2 \mathrm{D}$ behavior at sufficiently large $N_{s}$. The crossover value is given roughly by $N_{s}^{*} \approx r_{D}=D_{f} / D_{s}$, which corresponds to the average number of steps the walker must take before it takes a single step in the slow direction. More precisely, as is clear from the curvature of the data shown in Fig. 4, one cannot regard, for any degree of anisotropy, $N_{v}$ as being proportional to some power of $N_{s}$ and so should not expect perfect scaling or power-law behavior of $W_{f}$ with $D_{f}$. Hence the numbers given above for $\alpha$ must be regarded as approximations based on an estimate of the relevant local behavior of $N_{v}$ as a function of $N_{s}$, that behavior being determined by the length of a typical walk before a particle is absorbed. The arrows at the bottom Fig. 4 indicate $N_{s}^{*}$ for $r_{D}=99(\bullet), 999$ $(\square)$, and $9999(\square)$, and appear to predict well where these datasets show a distinct change in slope. We suggest the following physical explanation for this crossover behavior: Initially (for $N_{s}<N_{s}^{*}$ ) the walker is likely to remain on the same atomic row where it started, so $N_{v}$ is described well by the strict 1D limit, $N_{v} \sim N_{s}^{1 / 2}$. But once the walker takes a single step in the slow direction, it has access to a new row of unvisited sites and hence $N_{v}$ increases more rapidly than $N_{s}^{1 / 2}$. For $N_{s} \gg N_{s}^{*}$, the number of new atomic rows visited increases as $N_{s}$ as for an isotropic walker (albeit with a reduced prefactor) in two dimensions, and hence $N_{v}$ eventually scales as in the isotropic limit.

Returning to Fig. 3, we noted earlier that even for the



FIG. 5. The logarithm of $r_{w}$ divided by the logarithm of $r_{D}$ is shown as a function of $r_{D}$ for $D_{f}=1(\boldsymbol{\square}), 2(\square), 4(\boldsymbol{\bullet}), 8(\bigcirc), 16$ $(\boldsymbol{\Delta}), 32(\triangle), 64(\boldsymbol{\nabla}), 128(\nabla), 256(\diamond), 512(\diamond)$ in arbitrary units.

most highly anisotropic case simulated $\left(r_{D}=9999\right)$, the data do not quite reach the strict 1D scaling behavior. We believe that this is because the typical number of steps $N_{s}$ a walker must take before being incorporated in an island or reaching the wall is sufficiently large that $N_{v}$ is not strictly proportional to $N_{s}^{1 / 2}$ and so still shows remnants of $2 \mathrm{D}$ behavior. If the island density is around $10^{-3}$, which is typical, then some $10^{3}$ sites will be visited by a diffusing particle. From Fig. 4 one can see that the corresponding $N_{s}$, for $r_{D}$ $=9999$, is around $5 \times 10^{4}$, well beyond the number (about $10^{4}$ ) at which the crossover from one- to two-dimensional behavior occurs. Thus there are still remnants of $2 \mathrm{D}$ behavior present in the island nucleation process. In a real hightemperature growth experiment ${ }^{11}$ the typical number of steps taken by an adparticle is likely to be larger still, and hence we do not expect any nucleation-related quantity to be well described by true 1D behavior even for extremely anisotropic diffusion.

We describe next the behavior of the ratio $r_{w}=W_{f} / W_{s}$ as a function of $r_{D}=D_{f} / D_{s}$. In brief, we find that $r_{w}$ is not much different from $r_{D}^{1 / 2}$, independent of $D_{f}$. This point is simply made by plotting $\log \left(r_{w}\right) / \log \left(r_{D}\right)$ against $r_{D}$. Figure 5 is such a plot using ten values of $D_{f}$ (the same as the ones shown in Fig. 3) at each of four values of $r_{D}$ which are 4, 9 , 99 , and 999. The plot strongly supports the notion that $r_{w}$ is equal to $r_{D}^{\beta}$ where $\beta$ is close to $1 / 2$; the simulations actually give on average a value slightly larger than $1 / 2$, and the deviations from $1 / 2$ are typically larger as $r_{D}$ increases. In this limit $W_{s}$ is very small relative to the size of the cell and hence becomes difficult to determine with high precision from our simulations. Most of the data points in the figure have an uncertainty on the order of 0.01 which comes mainly from determining the average width of the denuded zone from many individual simulations. The uncertainty is somewhat larger for cases of large $r_{D}$ and small $D_{f}$ since then $W_{s}$ is particularly small and the relative uncertainty in $W_{s}$ correspondingly large.

We have done further simulations designed to test in cer- 




FIG. 6. For the case of extremely anisotropic (one-dimensional) islands, the width of the denuded-zone in the fast direction, in units of the total width of the system, is shown as a function of $D_{f}$ (arbitrary units) for diffusion anisotropies of $1(\square), 4(\square), 9(\bullet)$, $99(\bigcirc)$, and $999(\boldsymbol{\Delta})$. Lines of slope (on a log-log plot) $1 / 6$ and 1/4 are included for purposes of comparison.

tain respects how robust are the results just described. One such set of simulations employs anisotropic islands of finite size. Each mobile particle encountering an island is incorporated into the island, increasing its area by one lattice site. We have looked at two cases, one of which is to make the islands anisotropic with (on average) a linear size in the slow direction which is twice as large as that in the fast direction, reflecting approximately what is observed in experiments on $\mathrm{Si}(001)$ at relatively high temperature. The second, and more extreme, case is to make each island's size in the fast direction just one lattice site in extent while its length in the slow direction is equal to the number of particles in the island. This is closer to the observed low-temperature $\mathrm{Si}(001)$ behavior.

In both cases there is not much effect relative to what we have presented for point islands. Figure 6 shows $W_{f} / N$ vs. $D_{f}$ for the more extreme case of one-dimensional islands, with (from bottom to top) $r_{D}=1,4,9,99$, and 999. One can see from comparison with Fig. 3 (the various symbols correspond to the same $r_{D}$ in both figures and the straight lines in both figures are the same) that there is almost no qualitative consequence of allowing highly anisotropic islands. It is the case that for a given set of parameters, the denuded-zone widths are somewhat smaller for the 1D islands as compared with point islands reflecting the fact that point islands present smaller targets to the diffusing species, thereby allowing particles on average to travel farther before being absorbed.

Figure 7 displays $\log \left(r_{w}\right) / \log \left(r_{D}\right)$ against $r_{D}$ for ten values of $D_{f}$ in the limit of one-dimensional islands and may be compared directly with Fig. 5 which presents the same entities for point islands. One sees very little real difference between the two cases, which supports the contention that even very anisotropic islands do not give rise to results for $\beta$ much different from $1 / 2$. The uncertainty of the data points is again of the order of 0.01 as in Fig. 5 and it has the same tendency to be largest for large $r_{D}$ and small $D_{f}$.

The result $r_{w} \approx r_{D}^{1 / 2}$ is significantly different from the re-



FIG. 7. For the case of extremely anisotropic (one-dimensional) islands, the logarithm of $r_{w}$ divided by the logarithm of $r_{D}$ is shown as a function of $r_{D}$ for $D_{f}=1(\boldsymbol{\square}), 2(\square), 4(\boldsymbol{O}), 8(\bigcirc), 16(\mathbf{\Delta})$, $32(\triangle), 64(\nabla), 128(\nabla), 256(\diamond), 512(\diamond)$ in arbitrary units.

sult of Mo, et al., ${ }^{1}$ based on a dimensional analysis, which predicts an exponent of $\beta \approx 1 / 4$ (for extreme anisotropic diffusion) to $1 / 6$ (isotropic diffusion) instead of $1 / 2$. This has strong consequences for any attempt to extract the ratio $D_{f} / D_{s}$ from measurements of the denuded-zone widths. For example, if the measured ratio $r_{w} \approx 4$, we would conclude that $r_{D} \approx 16$ instead of being between about 250 and 4000 . We believe the origin of this discrepancy is that Mo, et al., ${ }^{1}$ apparently assumed a different adparticle lifetime (before incorporation into an island or a step) for fast diffusion as compared with slow diffusion. In fact for a given growth condition there is only a single adatom lifetime that depends on both diffusion constants (although it is actually dominated by fast diffusion for highly anisotropic cases). We also note that all of the simulations reported in Ref. 1 only had terrace boundaries perpendicular to the fast-diffusion direction (and periodic boundary conditions in the slow direction) and hence Mo et al. never actually simulated denuded zones in the slow direction.

We have done additional simulations exploring the consequences of anisotropic sticking coefficients since on $\mathrm{Si}(001)$ terraces the sticking probability at $S_{A}$ steps appears to be smaller than the sticking probability at the $S_{B}$ steps. ${ }^{3,12}$ This can be a factor for growth experiments on rectangular-shaped $\mathrm{Si}(001)$ terraces with $\langle 110\rangle$ oriented boundaries, in which case the fast- and slow-diffusion directions are perpendicular to a bounding $S_{A}$ and $S_{B}$ step, respectively. When the probability $p$ of a diffusing particle adhering to a wall, an island, or another mobile particle is different for a particle moving in the fast direction from that for a particle moving in the slow direction, anisotropy will be introduced into the denuded-zone widths quite independently ${ }^{3}$ of the difference between $D_{f}$ and $D_{s}$. We have looked at the case of $p=1$ in the slow direction but as small as 0.01 in the fast direction. In these simulations islands are treated as points to avoid confusing the consequences of anisotropic $p$ with those of anisotropic islands. However, both isotropic and anisotropic diffusion have been simulated. Further, we have looked at cases in which, in the fast direction, $p$ is the same for colli- 




FIG. 8. $r_{w}$ is plotted against the sticking coefficient $p$ in the $x$ direction for isotropic diffusion with a typical $D_{f}$; the sticking coefficient in the $y$ direction is unity; the points $(\bigcirc)$ are for reduced sticking only at the walls while points $(\square)$ are for reduced sticking for encounters with walls, islands, and other diffusing particles.

sions with walls as with islands or other diffusing particles and also cases in which $p$ is unity for collisions with islands and diffusing particles but less than unity for collisions with walls. Figure 8 displays $r_{w}$ as a function of $p$ for the two cases just described; $(\bigcirc)$ is for $p<1$ only for encounters with walls, and $(\square)$ is for $p<1$ for encounters with walls, islands, and diffusing particles. A typical (middle of the range) $D_{f}$ is used with $r_{D}=1$; the results are quite insensitive to the precise value of $D_{f}$. Because the island density profiles in the fast and slow directions do not have the same shape when $p \neq 1$, the denuded-zone widths depend on the criterion one employs to define the edge of the zone (for example, an island density $50 \%, 60 \%$, or $70 \%$ of the density at the terrace center). Hence $r_{W}$ is affected by this criterion; for a range of reasonable criteria, $r_{w}$ can vary by $\sim 0.1$ for the smaller values of $p$. Here, as elsewhere in the simulations, we used $60 \%$ as the criterion. One sees that $r_{w}$ decreases markedly as $p$ decreases and that there is not a huge difference between the two cases. One may conclude that if there is a reduction in $p$ in the fast direction, one should see a reduced value of $r_{w}$ as compared with the $p=1$ case. With regard to extracting the diffusion ratio $r_{D}$ from $r_{w}$, this means that the actual value of $r_{D}$ would be larger than one would estimate if isotropic sticking were assumed.

We can investigate the relative importance of anisotropic sticking as compared with anisotropic diffusion by looking at $r_{w}$ as a function of $r_{D}$ for various $p$. Figure 9 displays $r_{w}$ against $r_{D}$ for (from top to bottom) $p=0.5,0.2,0.1,0.05$, 0.02 , and 0.01 , with $p$ the same for particles encountering walls, islands, or other diffusing particles. The simulations use a single typical $D_{f}$ and are not sensitive to that value. The symbols show the simulation results. The cases with $p$ $\geqslant 0.1$ differ relatively little from what one finds for $p=1$, suggesting that so long as the sticking coefficient is not less than about 0.1 , one may obtain a reasonable value of $D_{f} / D_{s}$ from experimental measurements of the ratio $W_{f} / W_{s}$ by using the rule $D_{f} / D_{s} \approx\left(W_{f} / W_{s}\right)^{2}$. However, for $p<0.1$, Fig. 9

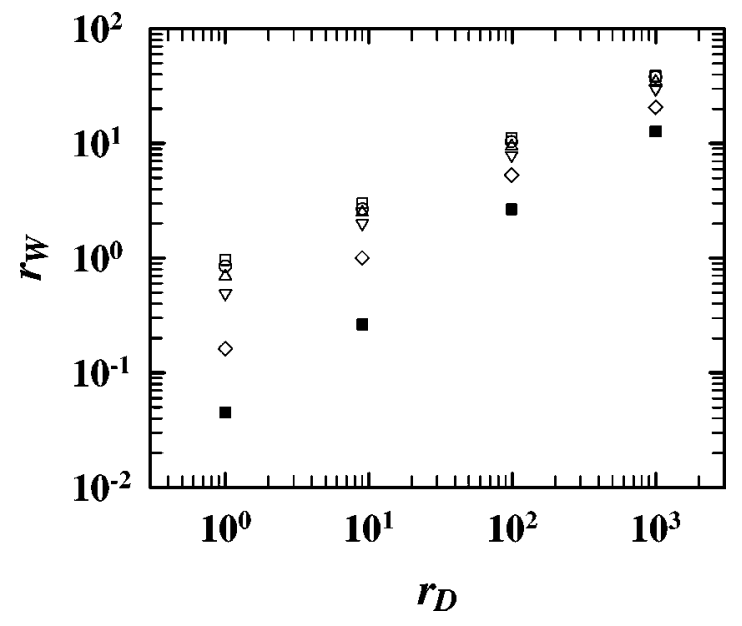

FIG. 9. $r_{w}$ is shown as a function of $r_{D}$ for a typical $D_{f}$ and with sticking coefficients in the fast direction of $0.5(\square), 0.2(\bigcirc), 0.1$ $(\triangle), 0.05(\nabla), 0.02(\diamond)$, and $0.01(\mathbf{\square})$.

shows that this rule will produce quite significant errors, and hence for systems where denuded zones are measured along two boundaries with highly anisotropic sticking, the rule $D_{f} / D_{s} \approx\left(W_{f} / W_{s}\right)^{2}$ will not be accurate.

With specific regard to $\mathrm{Si}(001)$, we note that the condition $p>0.1$ appears to be satisfied at relatively high temperatures ${ }^{13,14}\left(>640^{\circ} \mathrm{C}\right)$, where the step mobility $\Gamma$ for (global) $S_{A}$ and $S_{B}$ steps has been found to be nearly the same. This indicates that $S_{A}$ and $S_{B}$ steps have nearly the same sticking probability at these temperatures, since the step mobility is directly proportional to the step-edge attachment/detachment rate $\kappa$. In contrast, the condition $p$ $>0.1$ may not be satisfied for $\mathrm{Si}(001)$ at lower temperatures, where highly anisotropic sticking has been directly observed. ${ }^{3,12}$ In this case, the denuded-zone ratio $W_{f} / W_{s}$ can be used to estimate $D_{f} / D_{s}$ only if special care is taken (as was taken by Mo, et al. ${ }^{3}$ ) to compare only denuded zones along boundaries with similar sticking. This is possible to do on vicinal $\mathrm{Si}(001)$ surfaces with $\langle 110\rangle$ oriented miscut, provided the sticking at an "up-step" can be shown to be nearly the same as at a "down-step." But this is not possible to do with extremely large terraces formed by "flattening" lithographically defined craters, which are required to study the very large denuded zones that occur at elevated growth temperatures. Fortunately, as discussed above, sticking at $S_{A}$ and $S_{B}$ steps appears to be much more symmetric at elevated temperatures. ${ }^{13,14}$

\section{CONTINUUM MODEL OF THE FORMATION OF DENUDED ZONES}

We next consider denuded-zone formation at much higher temperature, where a critical island consists of several hundreds of adparticles. ${ }^{9}$ This nucleation condition is much harder to simulate with direct $\mathrm{MC}$ techniques, both because the simulations must be much longer to grow the necessarily large islands and because a larger cell should be used to accommodate numerous islands, each of which occupies several thousand sites. Consequently, in this section emphasis is 
on a continuum model of island nucleation and denudedzone formation, although some limited large-scale simulation results are also reported. The continuum model considers the spatial and temporal evolution of adparticle concentration and nucleation rather than tracking the motion of each adparticle. The main conclusion from this modeling is that the denuded-zone ratio scales with the diffusion constant ratio as $W_{f} / W_{s} \approx\left(D_{f} / D_{s}\right)^{\beta}$, with $\beta$ close to $1 / 2$ for a wide range of conditions; that is consistent with the results of our MC simulations.

The model is designed to closely match the experimental conditions of high-temperature $\left(530-700^{\circ} \mathrm{C}\right)$ nucleation and growth studied by Nielsen et al., ${ }^{11}$ who used low-energy electron microscopy (LEEM) to observe $\mathrm{Si}$ island nucleation on large $(>5 \mu \mathrm{m})$ step-free $\mathrm{Si}(001)-(2 \times 1)$ terraces prepared by the method proposed by Tanaka et al. ${ }^{15}$ A primary difference in the studies of Nielsen et al. from previous LEEM studies of $\mathrm{Si}$ island coarsening by Bartelt et al. ${ }^{16}$ is that Nielsen and co-workers observed nucleation and growth at constant temperature, whereas Bartelt and co-workers deposited $\mathrm{Si}$ at room temperature followed by coarsening at higher temperature $\left(670{ }^{\circ} \mathrm{C}\right)$.

Figure 10 shows a typical nucleation sequence on a $\sim 5 \mu \mathrm{m} \times 6 \mu \mathrm{m}$ rectangular terrace measured at $560{ }^{\circ} \mathrm{C}$ and using a $\mathrm{Si}$ deposition flux of $0.2 \mathrm{ML} / \mathrm{min}$. We note two main features from this sequence. First, at the time that islands start to be visible somewhat clearly to LEEM, Fig. 10(a), around $2 \mathrm{~s}$ after the start of growth, they appear almost simultaneously and uniformly across the central part of the terrace, leaving well-defined denuded zones around the terrace edges. (In reality, the actual island nucleation would occur before we could see the islands in LEEM because the islands whose sizes are close to critical islands are still too small to be visible due to the limitations on the spatial resolution of LEEM.) The denuded zones in the fast-diffusion direction are clearly larger than those in the slow direction [arrows in Fig. 10(b)]. During subsequent growth, the existing islands generally increase in size, but there is very little nucleation of new islands. These basic features were present ${ }^{11}$ for the same flux and at deposition temperatures ranging from $530{ }^{\circ} \mathrm{C}$ to $700{ }^{\circ} \mathrm{C}$

These observations suggest the following picture of island nucleation: After the Si flux is turned on at $t=0$, the adparticle concentration $c(x, y, t)$ increases uniformly over the terrace except close to the edges where it is suppressed by step-edge sticking. After $c(x, y, t)$ has reached a certain critical value (several percent above the equilibrium concentration $\left.^{9}\right)$ nucleation starts to occur. Since nucleation depends strongly ${ }^{9}$ on $c(x, y, t)$ and $c(x, y, t)$ is almost constant over the central part of the terrace, initial island nucleation occurs almost simultaneously throughout this region. Very soon after these islands appear and start to grow, they deplete surrounding areas of adparticles. Shortly thereafter (at a time we designate as $\tau_{\text {stop }}$ ) the island depletion zones start to overlap and quickly suppress new nucleation. The islands continue to grow as they absorb deposited $\mathrm{Si}$, but few new islands form.

To model and test this scenario, we assume a set of step and adparticle parameters, including $D_{f}$, which are appropri-
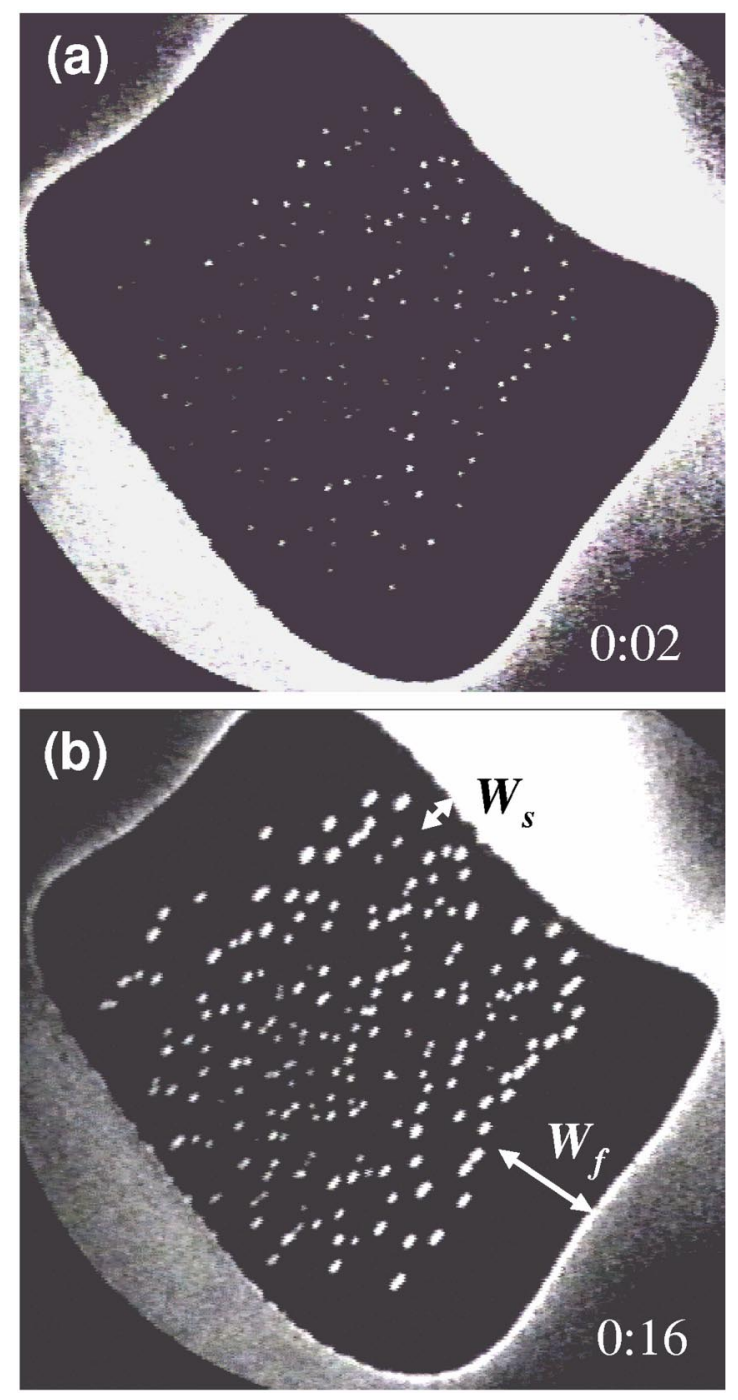

FIG. 10. LEEM images of island formation on a rectangular terrace $(\sim 6 \mu \mathrm{m} \times 5 \mu \mathrm{m})$ at (a) $2 \mathrm{~s}$ and (b) $16 \mathrm{~s}$, after starting $\mathrm{Si}$ deposition at $560{ }^{\circ} \mathrm{C}$ with a deposition rate of $0.2 \mathrm{ML} / \mathrm{min}$.

ate for a given experimental growth condition; these parameters are obtained from the literature. ${ }^{7-9,13,17}$ We then solve the time-dependent diffusion equation to model how the adparticle concentration $c(x, y, t)$ increases with time and then estimate the time-integrated island density $\omega(x, y, t)$ across the terrace using an expression for the island nucleation rate proposed by Theis and Tromp. ${ }^{9}$ The solution is obtained up to the time $\tau_{n}$ when the area-integrated total number of islands $\Omega(t)$ is equal to the measured number of islands for that growth condition. We then determine the size of the fast $\left(W_{f}\right)$ and slow $\left(W_{s}\right)$ denuded zones by finding the contour along which island density $\omega(x, y, t)$ is equal to a fixed fraction $\gamma$ (we tried $70 \%$ or $90 \%$ ) of the value at the center of the terrace. Finally, we compare $r_{w}=W_{f} / W_{s}$ to the diffusion constant ratio $r_{D}=D_{f} / D_{s}$. It is clear that a major assumption in this model is that the time $\tau_{n}$ corresponds reasonably to the time $\tau_{\text {stop }}$ when nucleation is strongly suppressed. For an extremely wide range of assumed parameter values (giving a range of values for $\tau_{n}$ ) we always find $W_{f} / W_{s}$ $\approx\left(D_{f} / D_{s}\right)^{\beta}$, with $\beta$ close to $1 / 2$. This general conclusion is 
TABLE I. Model parameter values at $560{ }^{\circ} \mathrm{C}$ referred to the literature in the table; $R=3.5 \times 10^{-3} \mathrm{ML} / \mathrm{s}$.

\begin{tabular}{lcccc}
\hline \hline$c_{e q}\left(\mathrm{~nm}^{-2}\right)$ & $D_{f}\left(\mathrm{~nm}^{2} / \mathrm{s}\right)$ & $\beta(\mathrm{eV} / \mathrm{nm})$ & $\Gamma\left(\mathrm{nm}^{3} / \mathrm{s}\right)$ & $\kappa_{A} \approx \kappa_{B}(\mathrm{~nm} / \mathrm{s})$ \\
\hline 0.02587 (Ref. 17) & $6.1 \times 10^{5}$ (Refs. 7 and 8) & 0.072 (Ref. 13) & 19.60 (Refs. 9 and 13) & $8.71 \times 10^{3}$ (Ref. 9) \\
\hline \hline
\end{tabular}

further supported by several representative MC simulations we have done (see below) in the large-critical-cluster regime.

We now describe the model calculations in more detail. Prior to island nucleation, the adparticle concentration $c(x, y, t)$ is governed by a time-dependent diffusion equation, ${ }^{18,19}$

$$
D_{f} \frac{\partial^{2} c(x, y, t)}{\partial x^{2}}+D_{s} \frac{\partial^{2} c(x, y, t)}{\partial y^{2}}+R=\frac{\partial c(x, y, t)}{\partial t},
$$

where $D_{f}$ and $D_{s}$ are, as above, the diffusion constants of adparticles parallel (fast) and perpendicular (slow) to the surface dimer rows, respectively, and $R$ is the $S i$ deposition rate. Equation (1) is subject to the following boundary conditions at the terrace boundary: ${ }^{18,20}$

$$
\begin{gathered}
D_{f} \frac{\partial c(x, y, t)}{\partial x}=\mp \kappa_{A}\left[c\left( \pm L_{f} / 2, y, t\right)-c_{e q}\right], \\
D_{s} \frac{\partial c(x, y, t)}{\partial y}=\mp \kappa_{B}\left[c\left(x, \pm L_{s} / 2, t\right)-c_{e q}\right],
\end{gathered}
$$

where $\kappa_{A, B}$ is the adparticle attachment rate at $S_{A, B}$ step edges, and $c_{e q}$ is the equilibrium adparticle concentration. The geometry of the terrace is roughly rectangular, the terrace size in the fast direction $\left(L_{f}\right)$ is $\approx 6 \mu \mathrm{m}$ and that in the slow direction $\left(L_{s}\right)$ is $\approx 5 \mu \mathrm{m}$. The boundary condition balances the net diffusion current toward the step edge with the net rate at which adparticles attach to the step edge.

The nucleation rate of stable islands based on homogeneous nucleation theory has been given ${ }^{9,21}$ as

$$
\omega(x, y, t)=2 \sqrt{\pi N} \frac{\Gamma}{a^{3} k_{B} T} c e^{-\beta a \sqrt{\pi N}}
$$

which we rewrite as

$$
\omega(x, y, t)=\frac{2 \beta \pi \Gamma}{a^{2} k_{B} T}\left(\frac{c}{\ln \left(c / c_{e q}\right)}\right) \exp \left[-\frac{A}{\ln \left(c / c_{e q}\right)}\right],
$$

where $A$ is $\left(a \beta \sqrt{\pi} / k_{B} T\right)^{2}, a^{2}=0.29 \mathrm{~nm}^{2}$ is the squared lattice spacing for a diffusing adparticle, assumed here to be a dimer, ${ }^{6,7,17,19} \mathrm{~N}$ is the number of dimers in a critical nucleus, $\Gamma$ is the step mobility, which is related to the step attachment rate by ${ }^{18} \kappa=\Gamma /\left(a^{4} c_{e q}\right)$, and $\beta$ is the step-free energy.

Table I lists the parameters used in this modeling for an assumed temperature $T=560^{\circ} \mathrm{C}$. All these parameters are temperature dependent. For the model results shown in Figs. 11-13, we have fixed the fast diffusion constant ${ }^{8} D_{f}$ $\cong 6.1 \times 10^{5} \mathrm{~nm}^{2} / \mathrm{s}$, and then varied $D_{s}$ so that $r_{D}$ assumes the values of $1,10^{1 / 2}, 10,10^{3 / 2}, 10^{2}, 10^{5 / 2}$, and $10^{3}$.
Figure 11 shows how the total island number $\Omega(t)$ varies with the deposition time for $r_{D}=10^{3 / 2}$. We see that $\Omega(t)$ increases very abruptly after the "first" island nucleates at $t \cong 0.11 \mathrm{~s}$ until $\Omega(t) \cong 177$ (the measured number of islands at $560{ }^{\circ} \mathrm{C}$ at $t \cong 0.17 \mathrm{~s}$ ). These numbers somewhat correspond to the experiment, where the actual island nucleation was expected to occur well before it became visible to LEEM at $t \cong 2 \mathrm{~s}$.

Figure 12 shows profiles of the adparticle concentration $c(x, y, t)$ and the island density $\omega(x, y, t)$ in the (a) fast and (b) slow directions for $r_{D}=10^{3 / 2}$, at the time $t=\tau_{n}$ when nucleation is assumed to stop. We see that both $c(x, y, t)$ and $\omega(x, y, t)$ are roughly constant around the center of the terrace, and are suppressed at the terrace edges with longerrange suppression in the fast-diffusion direction. However, the suppression is significantly stronger for $\omega(x, y, t)$ than for $c(x, y, t)$. This is due to the strong dependence on adparticle concentration of the island nucleation rate as shown in Eq. (5). Our modeling is consistent with the experimental observation of relatively distinct denuded-zone boundaries. The arrows in Figs. 12(a) and 12(b) indicate the fast and slow denuded-zone widths, respectively, assuming a $70 \%$ criterion for the denuded-zone boundary.

Figure 13 shows the relation between the ratio of the denuded-zone widths and the ratio of the diffusion constants obtained from measuring the denuded-zone widths for different values of $r_{D}$. Our finding is consistent with the relation $r_{w}=r_{D}^{\beta}$ where $\beta$ is very nearly $1 / 2$. To see whether or not the arbitrariness in choosing any particular value of island density to define the denuded-zone width affects the exponent $\beta$, we use two different fractions $70 \%$ and $90 \%$ for this purpose.



FIG. 11. Number of stable islands depending on deposition time at $560{ }^{\circ} \mathrm{C}$ for $r_{D}=10^{3 / 2}$; it reaches one around $0.11 \mathrm{~s}$ and $\sim 177$ (the number observed in the experiment) around $0.17 \mathrm{~s}$ after starting $\mathrm{Si}$ deposition. 



FIG. 12. Adparticle concentrations with respect to equilibrium adparticle concentration (dotted lines) and stable island densities with respect to the terrace center density (solid lines) in the (a) fast and (b) slow directions at $560{ }^{\circ} \mathrm{C}$ for $r_{D}=10^{3 / 2}$ when the island number reaches $\sim 177$ observed in experiment; the arrows represent the denuded zones in both directions based on a $70 \%$ criterion for the denuded-zone boundary.

Although the denuded-zone width itself in each direction definitely depends on the choice, the ratio of the denudedzone widths in the fast and slow directions is quite insensitive to it.

In Fig. 14 we show as solid discs the locations of large islands (ones that grow indefinitely when there is a rain of atoms on the surface) found from a MC simulation of a system measuring $4000 \times 4000$ sites. The simulation is much like the ones described in Sec. II except that now diffusing



FIG. 13. $r_{w}$ vs $r_{D}$ at $560{ }^{\circ} \mathrm{C}$ : The slope (on a log-log plot) is $0.48 \pm 0.002$.

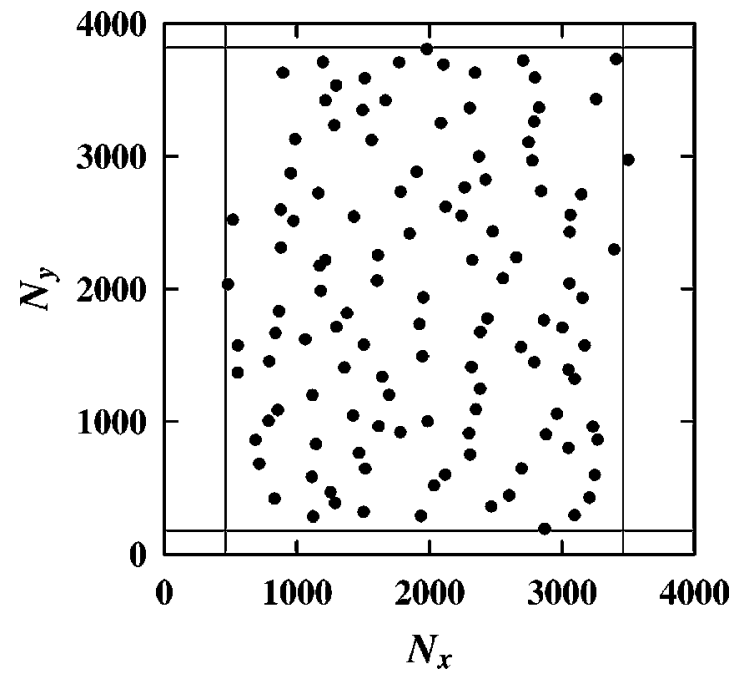

FIG. 14. The island distribution in a system measuring $4000 \times 4000$ sites with $r_{D}=9$. The lines represent a best fit to the denuded-zone edges using a ratio $r_{w}=3$. The critical cluster size in this simulation is on the order of several hundred particles.

particles are spawned by the walls and by the islands themselves. There are numerous new parameters entering these simulations; they have been chosen so that in equilibrium (with $R=0$ ) the fraction of occupied sites on the terrace is on the order of $1 / 2$ of a percent and also so that in the presence of a rain of atoms, the critical cluster size is at least several hundred to one thousand particles. There is no single critical cluster size because whether any given island grows indefinitely or evaporates depends to some extent on its immediate environment. The discs do not represent the sizes of the islands which range from one thousand to several thousand particles. The islands are not actually isotropic in shape but are rather some two times as large in the slow direction as in the fast direction, a shape obtained by simply rearranging particles once they are added to an island and chosen to replicate the shape observed in experiments at elevated temperatures. ${ }^{19}$ For this particular simulation, $r_{D}=9$. One can see clearly the denuded zones in the fast and slow directions. The solid lines represent a fit to the edges of the denuded zones assuming the width in the fast direction is three times as large as that in the slow direction. One may conclude that the figure supports the rule $r_{w} \approx r_{D}^{1 / 2}$. Additional simulations with $r_{D}=1$ and $r_{D}=4$ have been done and also support this conclusion.

Physically, the relation $r_{w} \approx r_{D}^{1 / 2}$ makes good common sense. As discussed above (and previously ${ }^{1}$ ) denuded-zone formation is related to a characteristic maximum adparticle lifetime $\tau_{n}$ before it is incorporated into a step or an existing island. This physically corresponds to the moment when nucleated islands on the terrace become sufficiently numerous and large as to soak up diffusing adparticles, effectively suppressing the adparticle concentration and hence also new island nucleation. During this time, an adparticle can diffuse characteristic distances $\sqrt{D_{f} \tau_{n}}$ and $\sqrt{D_{s} \tau_{n}}$ in the fast- and slow-diffusion directions, respectively. If an adparticle encounters a step during this time, it may attach to the step and hence reduce the local adparticle concentration. Conse- 
quently, there will be "zones" of reduced adparticle concentration (and hence reduced nucleated island density) of approximate width $W_{f} \propto \sqrt{D_{f} \tau_{n}}$ and $W_{s} \propto \sqrt{D_{s} \tau_{n}}$ bounding steps that are oriented perpendicular to the fast- and slow-diffusion directions, respectively. Hence the ratio of denuded zones is approximately $r_{W}=W_{f} / W_{s} \approx\left(D_{f} / D_{s}\right)^{1 / 2}$. Consistently with earlier discussion, this approximate relation only breaks down if sticking at step edges is highly anisotropic, since this would also affect the relative sizes of $W_{f}$ and $W_{s}$, quite independently of $D_{f}$ and $D_{s}$.

We note that a similar relation was also assumed by Doi et al. ${ }^{22}$ who measured the annealing-induced extension of denuded zones in the fast and slow directions on $\mathrm{Si}(001)$, as a function of sample annealing time $t_{\text {anneal }}$ after depositing $\mathrm{Si}$ at room temperature. They observed anisotropic annealing-induced denuded zones, which followed the approximate relation $W\left(t_{\text {anneal }}\right) \approx \sqrt{t_{\text {anneal }}}$ in both the fast and slow directions. They then assumed that $W_{f, s}\left(t_{\text {anneal }}\right)$ $\approx \sqrt{D_{f, s} t_{\text {anneal }}}$ in each direction, from which follows the relation $W_{f} / W_{s} \approx\left(D_{f} / D_{s}\right)^{1 / 2}$.

\section{SUMMARY}

By using MC simulations and a continuum model, we have studied how the formation of denuded zones is related to adparticle diffusion during growth on surfaces with anisotropic diffusion. In the limit of small critical clusters consisting of just two adparticles (low-temperature), our MC simulations demonstrate that the relative size of denuded zones in the fast- and slow-diffusion directions is proportional to the ratio of diffusion constants raised to a power close to $1 / 2$, i.e., $W_{f} / W_{s} \approx\left(D_{f} / D_{s}\right)^{\beta}, \beta \approx 1 / 2$. The same relation was obtained in the large-critical-cluster limit (high temperature)

*Email address: jpelz@mps.ohio-state.edu

${ }^{1}$ Y.-W. Mo, J. Kleiner, M.B. Webb, and M.G. Lagally, Surf. Sci. 268, 275 (1992).

${ }^{2}$ Y.-W. Mo, J. Kleiner, M.B. Webb, and M.G. Lagally, Phys. Rev. Lett. 66, 1998 (1991).

${ }^{3}$ Y.-W. Mo and M.G. Lagally, Surf. Sci. 248, 313 (1991).

${ }^{4}$ G. Brocks, P.J. Kelly, and R. Car, Phys. Rev. Lett. 66, 1729 (1991).

${ }^{5}$ D. Dijkkamp, E.J. van Loenen, and H.B. Elswijk, in Ordering at Surfaces and Interfaces, edited by A. Yoshimore, T. Shinjo, and H. Watanobe (Springer, Berlin, 1992), p. 85.

${ }^{6}$ R.A. Wolkow, Phys. Rev. Lett. 74, 4448 (1995).

${ }^{7}$ M. Krueger, B. Borovsky, and E. Ganz, Surf. Sci. 385, 146 (1997).

${ }^{8}$ B.S. Swartzentruber, Phys. Rev. Lett. 76, 459 (1996).

${ }^{9}$ W. Theis and R.M. Tromp, Phys. Rev. Lett. 76, 2770 (1996).

${ }^{10}$ A. Pimpinelli, J. Villain, and D.E. Wolf, Phys. Rev. Lett. 69, 985 (1992).

${ }^{11}$ J.-F. Nielsen, K.-B. Park, J.P. Pelz, H. Hibino, C.-W. Hu, and I.S.T. Tsong (unpublished). from a continuum model based on homogeneous nucleation theory. ${ }^{9,21}$ This relation supplies a relatively simple way to extract diffusion anisotropy from the denuded zones measurable in epitaxial growth experiments. We also examined the relation between the number of steps $N_{s}$ in a random walk and the number of sites visited $N_{v}$ in that walk. This relation determines in part how denuded zone widths scale with the diffusion constant and diffusion anisotropy in the scaling argument introduced by Mo et al. ${ }^{1}$ Interestingly, in the case of anisotropic diffusion the relation between $N_{s}$ and $N_{v}$ displays a crossover behavior from strict $1 \mathrm{D}$ to $2 \mathrm{D}$ diffusion as $N_{s}$ increases rather than showing a behavior intermediate between the two limiting cases, suggesting that the apparent scaling exponent relating $W$ and $D$ depends on the typical number of steps an adparticle makes before meeting an existing island, another adparticle, or a step edge. With this analysis, we could also explain an $\approx 15 \%$ discrepancy between the scaling exponent relating $W$ to $D$ between values obtained from the MC simulations and those predicted in the scaling argument for 2D isotropic diffusion. Finally, we investigated the effect of anisotropic sticking of adparticles to step edges on denuded-zone formation and anisotropy. It turned out that as long as the smaller probability of sticking is not less than about 0.1 , we could use the relation $W_{f} / W_{s}$ $\approx\left(D_{f} / D_{s}\right)^{1 / 2}$ to obtain $D_{f} / D_{s}$ with a relatively small error.

\section{ACKNOWLEDGMENTS}

We wish to thank Ralf Bundschuh for useful information concerning two-dimensional random walks and $\mathrm{H}$. Hibino, C.-W. Hu, I. S. T. Tsong for helpful discussions and for allowing us to use their LEEM data (Fig. 10). This work was supported by NSF Grant No. DMR-0074416.

${ }^{12}$ A.J. Hoeven, J.M. Lenssinck, D. Dijkkamp, E.J. van Loenen, and J. Dieleman, Phys. Rev. Lett. 63, 1830 (1989).

${ }^{13}$ N.C. Bartelt, R.M. Tromp, and E.D. Williams, Phys. Rev. Lett. 73, 1656 (1994).

${ }^{14}$ N.C. Bartelt and R.M. Tromp, Phys. Rev. B 54, 11731 (1996).

${ }^{15}$ S. Tanaka, C.C. Umbach, J.M. Blakely, R.M. Tromp, and M. Mankos, Appl. Phys. Lett. 69, 1235 (1996).

${ }^{16}$ N.C. Bartelt, W. Theis, and R.M. Tromp, Phys. Rev. B 54, 11741 (1996).

${ }^{17}$ R.M. Tromp and M. Mankos, Phys. Rev. Lett. 81, 1050 (1998).

${ }^{18}$ H.-C. Jeong and E.D. Williams, Surf. Sci. Rep. 34, 171 (1999).

${ }^{19}$ J.-F. Nielsen, J.P. Pelz, H. Hibino, C.-W. Hu, and I.S.T. Tsong, Phys. Rev. Lett. 87, 136103 (2001).

${ }^{20}$ W.K. Burton, N. Cabrera, and F.C. Frank, Philos. Trans. R. Soc. London, Ser. A 243, 299 (1951).

${ }^{21}$ J.W. Christian, The Theory of Transformations in Metals and Alloys (Pergamon, New York, 1975).

${ }^{22}$ T. Doi, M. Ichikawa, S. Hosoki, and K. Ninomiya, Surf. Sci. 357-358, 868 (1996). 